\title{
3D-printed Ti6Al4V scaffolds combined with pulse electromagnetic fields enhance osseointegration in osteoporosis
}

\author{
MINGFU YE ${ }^{1}$, WENJUN LIU ${ }^{1}$, LIHUI YAN ${ }^{1}$, SHAOLONG CHENG $^{1}$, XIAOXIONG LI $^{2}$ and SHICHONG QIAO ${ }^{3}$ \\ ${ }^{1}$ Department of Oral Implantology, Xiamen Key Laboratory of Stomatological Disease Diagnosis and Treatment, \\ Stomatological Hospital of Xiamen Medical College, Xiamen, Fujian 361008; ${ }^{2}$ Department of Pain, Renji Hospital, \\ Shanghai Jiaotong University School of Medicine, Shanghai 201112; ${ }^{3}$ Department of Implant Dentistry, \\ Shanghai Ninth People's Hospital, Shanghai Jiaotong University School of Medicine, Shanghai 200011, P.R. China
}

Received October 27, 2020; Accepted March 8, 2021

DOI: $10.3892 / \mathrm{mmr} .2021 .12049$

\begin{abstract}
The loosening and displacement of prostheses after dental implantation and arthroplasty is a substantial medical burden due to the complex correction surgery. Three-dimensional (3D)-printed porous titanium (pTi) alloy scaffolds are characterized by low stiffness, are beneficial to bone ingrowth, and may be used in orthopedic applications. However, for the bio-inert nature between host bone and implants, titanium alloy remains poorly compatible with osseointegration, especially in disease conditions, such as osteoporosis. In the present study, 3D-printed pTi scaffolds with ideal pore size and porosity matching the bone tissue, were combined with pulse electromagnetic fields (PEMF), an exogenous osteogenic induction stimulation, to evaluate osseointegration in osteoporosis. In vitro, external PEMF significantly improved osteoporosis-derived bone marrow mesenchymal stem cell proliferation and osteogenic differentiation on the surface of pTi scaffolds by enhancing the expression of alkaline phosphatase, runt-related transcription factor-2, osteocalcin, and bone morphogenetic protein-2. In vivo, Microcomputed tomography analysis and histological evaluation indicated the external PEMF markedly enhanced bone regeneration and osseointegration. This novel therapeutic strategy has potential to promote osseointegration of dental implants or artificial prostheses for patients with osteoporosis.
\end{abstract}

\section{Introduction}

Osteoporosis is a systemic osteopathy defined by insufficiency in bone repair due to the impairment of bone microstructure, the decrease of bone quality and density and

Correspondence to: Dr Shichong Qiao, Department of Implant Dentistry, Shanghai Ninth People's Hospital, Shanghai Jiaotong University School of Medicine, 639 Zhizaoju Road, Shanghai 200011, P.R. China

E-mail: shichong_qiao@hotmail.com

Key words: 3D-printed, porous titanium alloy, pulse electromagnetic field, osseointegration, osteoporosis the increase of bone fragility $(1,2)$. Moreover, the osteoporotic microenvironment is not conducive to the proliferation and osteogenic differentiation of bone marrow mesenchymal stem cells (BMSCs), causing excessive bone loss and decreased osteogenic capacity $(3,4)$. These factors lead to inadequate osseointegration within the bone and implant surfaces, thus causing an increased risk of complications in patients after joint replacement and dental implantation, due to implant loosening and displacement (5-7). Therefore, improving the osseointegration efficiency of implants would be of high clinical benefit to solve these problems.

Titanium (Ti) alloy is a widely applied material for orthopedic or dental implants with promising application prospects due to its predominant mechanical strength and corrosion resistance; however, it is restricted by its high stiffness which consequently results in stress-shielding-induced osteolysis $(8,9)$. Three-dimensional (3D) printing technology is a promising method for the generation of individualized implants with complex components and porous sections in a single process $(10,11)$. Interconnected porous implants with controlled pore size and porosity can significantly decrease the stiffness, imitate the structure of natural bone tissue superiorly and promote bone regeneration. Moreover, bone ingrowth into the micropores of 3D-printed porous $\mathrm{Ti}(\mathrm{pTi})$ alloy could enhance the osseointegration and improve the stability of the implants (12-14). However, considering the biological inertia, smooth surface and poor cellular adhesion of Ti alloys, pTi scaffolds may fail due to insufficient osteointegration with the surrounding bone, especially under pathological states, such as osteoporosis $(15,16)$. Therefore, improving the bioactivity of osteoblast-related cells on the surface of titanium alloy is anticipated to treat the postoperative complications of osteoporosis patients. Bai et al (17) constructed a pTi/poloxamer 407 hydrogel system loaded with zoledronate, a bisphosphonate, thus obtaining an optimized osseointegration effect in osteoporotic defect models. In addition, Vladescu et al (10) coated 3D-printed Ti6Al4V scaffolds with HA, and calcium phosphate nanoparticles to improve the bioactivity and biocompatibility of the scaffolds. However, as it is difficult to release the loaded drugs continuously and maintain the stability of bio-coating, implanting pTi scaffolds into bone defects followed by extracorporeal non-invasive and repetitive 
therapy may be a potential strategy to promote bone integration $(18,19)$.

Pulse electromagnetic fields (PEMF), which are transient electromagnetic fields produced in a coil when a pulse current generated by a pulse generator passes through the coil, are considered as a non-invasive treatment with the effect of promoting bone regeneration in clinical applications (20). In terms of cytology, external PEMF have could promote the early and late osteogenesis, enhance mineralization of BMSCs (21), improve cell viability and enhance alkaline phosphatase (ALP) activity of osteoblasts (22), as well as inhibit bone resorption by inhibiting the formation and maturation of osteoclasts (23). Although previous studies have indicated that PEMF therapy has beneficial effects on bone regeneration and significantly enhances the healing of fracture, non-union and other orthopedic diseases, it has not been widely used to enhance osteointegration for osteoporotic bone defects $(24,25)$.

In the present study, it was hypothesized that the combination therapy of 3D-printed pTi and PEMF may have a positive effect on bone regeneration in vitro and in vivo in osteoporotic bone defects. The pTi implant supports optimized pore size and porosity, which match the mechanical properties of the bone tissue to decrease stress shielding. Additionally, a previous study has demonstrated that PEMF therapy $(50 \mathrm{~Hz}$; $1 \mathrm{mT}$ ) could induce osteogenic differentiation and proliferation of MSCs (26). In the present study, PEMF were used at the same frequency and intensity to detect whether PEMF therapy affect the fate of osteoporosis-derived BMSCs (OP-BMSCs) and enhance osseointegration in osteoporotic rabbits (Fig. 1). To the best of the authors' knowledge, this is the first study to investigate the combination of 3D-printed pTi and PEMF to promote osseointegration in osteoporosis to reduce complications following implantation.

\section{Materials and methods}

Materials. Ti6Al4V powder was purchased from AK Medical Co., Ltd. Low glucose Dulbecco's Modified Eagle's Medium (LG-DMEM), streptomycin-penicillin and fetal bovine serum (FBS) were obtained from Gibco, Thermo Fisher Scientific, Inc. Cell Counting Kit-8 (CCK-8; cat. no. C0039) and Calcein acetoxymethyl ester (Calcein-AM)/Propidium Iodide (PI; cat. no. C2015M) were purchased from Beyotime Institute of Biotechnology. Rhodamine-phalloidin and DAPI were supplied by Invitrogen (Thermo Fisher Scientific, Inc.). The media for alizarin red staining (ARS) and osteogenic differentiation of rabbit BMSCs (cat. no. RBXMX-90021) were purchased from Cyagen Biosciences. The $4 \%$ paraformaldehyde solution and PBS were obtained from Beijing Solarbio Science \& Technology. Eastep Super Total RNA Extraction Kit was supplied by Promega Corporation. Perfect Real Time RT reagent kit, Prime Script RT reagent kit, and SYBR Premix Ex Taq II kit were provided by Takara Biotechnology. TRIzol ${ }^{\circledR}$ reagent was supplied by Invitrogen (Thermo Fisher Scientific, Inc.). Masson stains were obtained from Thermo Fisher Scientific, Inc. The estrogen ELISA kit (cat. no. ML-Elisa-1434) was obtained from R\&D Systems.

Preparation of pTi implants. Ti6Al4V porous scaffolds conducted by $3 \mathrm{D}$ printing were prepared by using additive manufacturing with an electron beam melting system (Q10, Arcam AB), as previously reported $(27,28)$. Briefly, pTi alloy implants (porosity, $70 \%$; pore size, $600 \mu \mathrm{m}$ ) with a pre-designed 3D template were translated to a standard triangulation language (STL) file, which was loaded into the EBM system. Spherical Ti alloy powder was melted layer by layer according to the preset parameters (porosity, 70\%; pore size, $600 \mu \mathrm{m}$ ), then solidified by cooling. Disk-shaped ( $\varphi 10 \mathrm{~mm}$ x L3 mm) pTi implants for microstructure identification in vitro experiments, and columnar-shaped ( $\varphi 6 \mathrm{~mm}$ x L10 mm) pTi implants for osseointegration study in vivo. All prepared pTi implants were ultrasonically cleaned and sequentially cleaned in acetone, ethyl alcohol and deionized water for $\sim 15 \mathrm{~min}$ for each treatment.

Characterization of pTi implants. To verify whether the prepared implants match with the pre-designed parameters, the porosity of samples was detected by micro-computed tomography (CT) (SkyScan 1076 scanner, Bruker micro-CT NV) and analyzed using NRecon software (version 1.6.6; Bruker micro-CT). To calculate the average pore size and distribution, the microstructure of pTi scaffolds was obtained using a JSM-6700F scanning electron microscope (SEM; JEOL, Ltd.), and images were quantitatively analyzed using ImageJ $1.50 \mathrm{i}$ (National Institutes of Health).

Establishment of osteoporosis models. The osteoporotic rabbit model was generated by bilateral OVX. Five-month-old female New Zealand White rabbits $(n=30 ; 2.5 \mathrm{~kg})$ were randomly separated into two groups, the OVX group $(n=27)$ and the sham group $(n=3)$. Surgical operations were conducted under general anesthesia using $3 \%(\mathrm{w} / \mathrm{v})$ pentobarbital $(50 \mathrm{mg} / \mathrm{kg})$ intravenously. The median incision of the lower abdomen was chosen for surgery after skin shaving and sterilizing. The rabbits in the OVX group underwent bilateral OVX surgery, whereas those in the sham group were pseudo-operated, closing to the abdominal cavity after leaving the ovaries in situ. All rabbits were maintained in a cage individually and fed with standard chow $\left(15-25^{\circ} \mathrm{C}\right.$, humidity $60-70 \%, 12$-h light/dark cycle, fed twice a day, and drank ad libitum). Penicillin $(1.5 \mathrm{mg} / \mathrm{kg})$ was administered to post-operative rabbits by intramuscular injection for three consecutive days to prevent infection.

Ten months after surgery, the concentration of serum estrogen was measured using an ELISA kit. Furthermore, three animals from each group were sacrificed by intravenous injection of air under anesthesia by $3 \%(\mathrm{w} / \mathrm{v})$ pentobarbital $(150 \mathrm{mg} / \mathrm{kg})$ intravenously, and distal femurs were harvested for micro-CT assessment to confirm the establishment of osteoporosis.

Scaffold implantation and PEMF treatment. At 10 months post-OVX, osteoporotic rabbits were used in vivo osseointegration experiments. After general anesthesia using 3\% (w/v) pentobarbital $(50 \mathrm{mg} / \mathrm{kg})$ and preparation for operation, a longitudinal incision was performed in the distal femur of the left hind limb. After exposing the bony surface of the lateral condyle, cylindrical bone defects with a diameter of $6 \mathrm{~mm}$ and a depth of $10 \mathrm{~mm}$ were prepared with a bone drill. The defects were transplanted with pTi implants, then the incisions were closed by absorbable sutures. Postoperative management was carried out as aforementioned. 


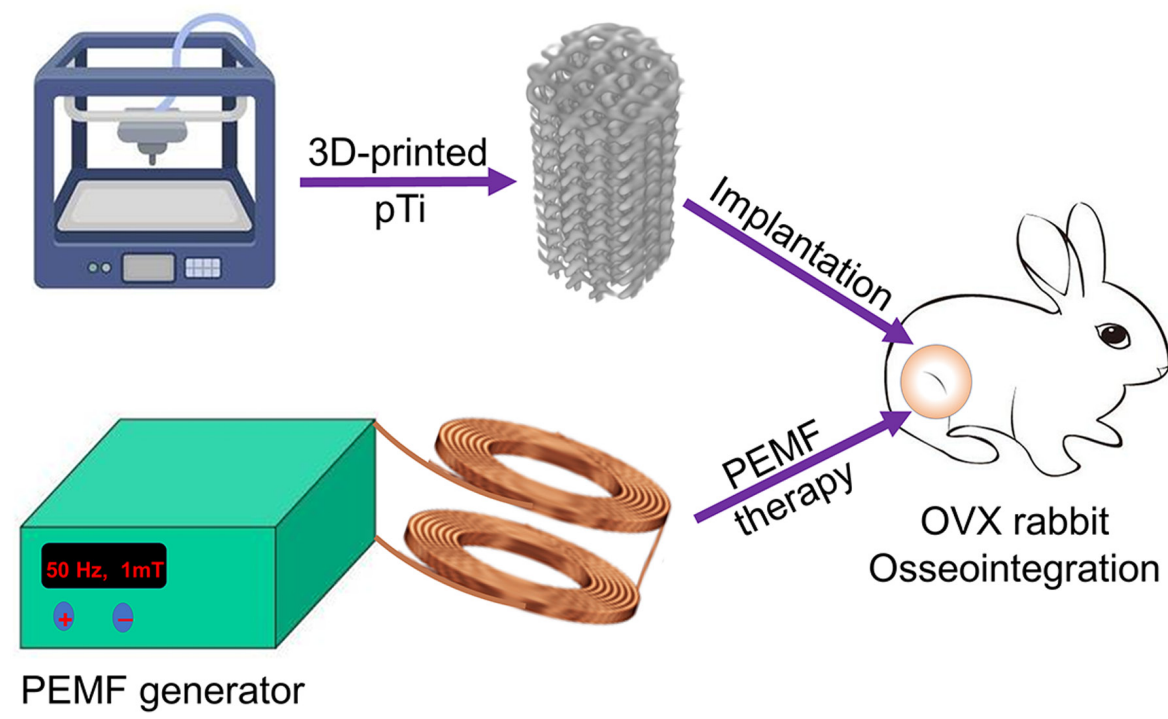

Figure 1. Combination therapy of 3D-printed pTi and PEMF to promote osseointegration in osteoporosis. The osteoporosis model was established by bilateral OVX in rabbits. The 3D-printed pTi was implanted into the distal femur of osteoporosis rabbits and then given extracorporeal PEMF treatment. pTi, porous titanium; PEMF, pulse electromagnetic field; OVX, ovariotomy.

On the fourth day after the implantation, the 24 osteoporotic rabbits were randomly divided into two groups: i) pT1 group, which received pTi implants without PEMF; and ii) pTi + PMEFs group, which received pTi implants with PEMF therapy. The rabbits of the pTi + PEMF group were kept in a plastic fixer, with their left legs placed within the scope of the coils of the PEMF machine in order to expose them to the pulse electromagnetic waves. The treatment parameters of PEMF were $50 \mathrm{~Hz}, 1 \mathrm{mT}, 2 \mathrm{~h}$ per day. After 6 and 12 weeks of treatment, rabbits were sacrificed by intravenous injection of pentobarbital sodium $100 \mathrm{mg} / \mathrm{kg}$, and femur samples were collected and fixed in $4 \%$ polyformaldehyde solution for further Micro-CT analysis and histological evaluation.

Isolation and culture of OP-BMSCs.OP-BMSCs were extracted from female rabbits $(\mathrm{n}=3) 10$ months after bilateral ovariotomy (OVX) and cultured as previously reported (27). Briefly, BMSCs derived from the OVX rabbits were harvested from the marrow cavity of long bone of extremities after centrifugation (996 x g, $20 \mathrm{~min}, 20^{\circ} \mathrm{C}$ ) and cultured in LG-DMEM medium containing $10 \% \mathrm{FBS}(\mathrm{v} / \mathrm{v})$ and $1 \%$ penicillin and streptomycin in a humidified environment with $37^{\circ} \mathrm{C}$ and $5 \% \mathrm{CO}_{2}$. Once OP-BMSCs grew to $~ 80 \%$ confluence, the adherent cells were treated with $0.25 \%(\mathrm{w} / \mathrm{v})$ trypsin/EDTA at $37^{\circ} \mathrm{C}$ for $3 \mathrm{~min}$. The cell suspension was collected and centrifuged ( $377 \mathrm{x} \mathrm{g}, 5 \mathrm{~min}$, $20^{\circ} \mathrm{C}$ ), then the obtained cell precipitates were resuspended and passaged. The third generation of OP-BMSCs was used in subsequent in vitro experiments.

In vitro cell experiments. To evaluate on cell attachment, proliferation, survival rate, morphology, and osteogenic differentiation of OP-BMSCs, CCK-8 assays, Calcein-AM/PI and rhodamine phalloidin staining, and reverse transcription quantitative-PCR (RT-qPCR) were carried out. Briefly, the pTi implants were immersed into a 24-well plate with DMEM, and OP-BMSCs $\left(2 \times 10^{5}\right.$ cells/well $)$ were added into each sample. The experiments consisted of two groups: i) pTi group, OP-BMSCs seeded on pTi implants under the basal culture medium without
PEMF; and ii) pTi + PEMF group, OP-BMSCs seeded on the pTi implants with PEMF therapy. The PEMF device (Prima PFM61009; Shanghai Prima Electronics Co., Ltd.) consisted of a generator and its connected coils. The OP-BMSCs in the pTi + PEMF group were placed on the center of the coils in an incubator and received PEMF stimulation during culture $(50 \mathrm{~Hz} ; 1 \mathrm{mT} ; 2 \mathrm{~h}$ per day). The medium was changed every 3 days.

Evaluation of cell viability. After incubating for $24 \mathrm{~h}$, the DMEM medium was discarded, and the CCK- 8 solution was then added into each well and incubated for $2 \mathrm{~h}$ at $37^{\circ} \mathrm{C}$ and $5 \% \mathrm{CO}_{2}$. The number of OP-BMSCs adhering to the surface of implants was quantitatively detected by measuring the absorbance at $450 \mathrm{~nm}$ using a microplate reader (Multiskan EX; Thermo Fisher Scientific, Inc.). To evaluate the cell proliferation, CCK- 8 assays were conducted after 1, 4 and 7 days of OP-BMSC culture on implants with or without PEMF. Live/Dead staining of the OP-BMSCs was detected at day 3 using a Calcein-AM/PI Double Stain kit according to the manufacturer's protocol, and observed under a FV1000 confocal laser scanning microscope (CLSM) (Olympus Corporation). For morphological evaluation at day 3 , the samples were fixed with $4 \%$ paraformaldehyde for $10 \mathrm{~min}$ at room temperature, then permeabilized by $0.1 \%$ Triton X-100 for $5 \mathrm{~min}$ at room temperature, and washed with PBS repeatedly for 3 times. Finally, the samples were stained with rhodamine-phalloidin for $30 \mathrm{~min}$ and DAPI for $5 \mathrm{~min}$ at room temperature in dark according to the manufacturer's instructions. The photomicrographs of stained cells were collected under a CLSM.

Evaluation osteogenic differentiation in vitro. To detect the osteogenic ability of OP-BMSCs in the presence of PEMF, basal culture medium was replaced by osteogenic induction medium including LG-DMEM along with $\beta$-glycerol-phosphate $(10 \mathrm{mM})$, ascorbate-2-phosphate $(50 \mu \mathrm{M})$, and dexamethasone $(0.1 \mu \mathrm{M})$. The protocols of cell culture and PEMF treatment were the same as the cell viability investigation in the previous 
Table I. Primer sequences.

\begin{tabular}{ll}
\hline Primer name & \multicolumn{1}{c}{ Oligonucleotide sequence (5'-3') } \\
\hline ALP-F & F: ATC GGA CCC TGC CTT ACC \\
ALP-R & R: CTC TTG GGC TTG CTG TCG \\
Runx-2-F & F: ACTACCAGCCACCGAGACCA \\
Runx-2-R & R: ACTGCTTGCAGCCTTAAATGACTCT \\
OCN-F & F: AGCCACCGAGACACCATGAGA \\
OCN-R & R: AGCCACCGAGACACCATGAGA \\
BMP-2-F & F: CAACACCGTGCTCAGCTTCC \\
BMP-2-R & R: TTCCCACTCATTTCTGAAAGTTCC \\
GAPDH-F & F: CAATGACCCCTTCATTGACC \\
GAPDH-R & R: TGGACTCCACGACGTACTCA
\end{tabular}

F, forward; R, reverse; ALP, alkaline phosphatase; Runx-2, runt-related transcription factor-2; OCN, osteocalcin; BMP-2, bone morphogenetic protein-2.

section. Following osteogenic culture for 14 or 21 days, ARS (15 min, room temperature) was conducted to assess calcium deposition according to the manufacturer's protocols and observed by a stereoscopic microscope (ZOOM-750; Tuming Optical Instrument Co., Ltd.). Subsequently, 10\% cetylpyridinium chloride was added into the dyed mineralized nodules to dissolve the stained calcium nodules for further semi-quantitative analysis. The obtained solution was detected at $450 \mathrm{~nm}$ through a microplate reader to assess unobservable calcium nodules inside the micropores.

Moreover, after treating with osteogenic induction medium for 14 and 21 days, the expression levels of alkaline phosphatase (ALP), runt-related transcription factor-2 (Runx-2), osteocalcin (OCN), and bone morphogenetic protein-2 (BMP-2) in OP-BMSCs in the different groups were measured using RT-qPCR. The sequences of primers were listed in Table I. Briefly, total RNA was extracted with TRIzol ${ }^{\circledR}$ (Thermo Fisher Scientific, Inc.) and $1 \mu \mathrm{g}$ total RNA per sample was reversely transcribed to cDNA using a Prime Script RT reagent kit according to the manufacturer's instructions. The expressions of target genes were quantified by RT-qPCR using the SYBR Premix Ex Taq II kit according to the manufacturer's protocol. The qPCR was conducted using $2 X$ Fast SYBR-Green Master Mix. Amplification was performed in 96-well optical reaction plates (Roche Diagnostics) on LightCycler 480 (Roche Diagnostics) using the following program: $94^{\circ} \mathrm{C}$ for $1 \mathrm{~min}$ to activate polymerase, 40 cycles at $94^{\circ} \mathrm{C}$ for $30 \mathrm{sec}, 57^{\circ} \mathrm{C}$ for $20 \mathrm{sec}$ and $72^{\circ} \mathrm{C}$ for $20 \mathrm{sec}$; melting curve analysis was performed after every run by heating up to $95^{\circ} \mathrm{C}$ to monitor presence of unspecific products. The relative mRNA expression levels were normalized to that of GAPDH and calculated using the $2^{-\Delta \Delta \mathrm{Cq}}$ method (29).

Micro-CT analysis. To explore the efficiency of bone formation, the samples were screened by micro-CT (voltage, $90 \mathrm{kV}$ voltage; current intensity, $114 \mathrm{~mA}$; pixel size, $18 \mu \mathrm{m}$ ). The cylindrical region of the pTi was targeted as the region of interest (ROI; a cylinder with diameter of $6 \mathrm{~mm}$ and height of $10 \mathrm{~mm}$ ) for $3 \mathrm{D}$ reconstruction and further parameter
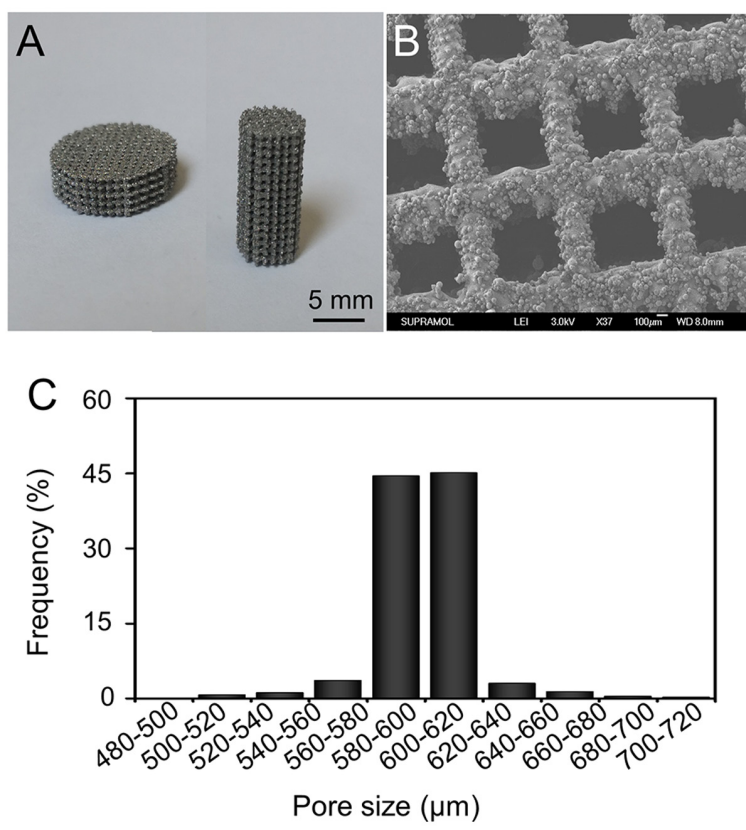

Figure 2. Characterization of 3D-printed pTi scaffolds. (A) Representative visual images of 3D-printed pTi implants. (B) Scanning electron microscope photomicrographs of 3D-printed pTi implants. (C) Pore size distribution of the pTi scaffolds. pTi, porous titanium.

analysis. Quantitative morphometric analysis, including bone volume/tissue volume ratio (BV/TV, \%), trabecular number (Tb.N, mm), trabecular thickness (Tb.Th, $\mathrm{mm}$ ) and trabecular separation (Tb.Sp, mm), was conducted using micro-CT auxiliary software (NRecon version 1.6.6).

Histological evaluation. The femur specimens containing pTi implants were embedded in methyl methacrylate, without undergoing decalcification and sectioned into thin sections (150-300 $\mu \mathrm{m}$ thickness). The sections were ground down and polished to 40-50 $\mu \mathrm{m}$ through the transverse saw cuts and polishing machine (EXAKT Apparatebau GmbH \& Co. KG). After polishing, these hard tissue sections were stained with Masson stain for $150 \mathrm{~min}$ at room temperature to evaluate the bone regeneration and osseointegration with the porous implants.

Statistical analysis. All data are presented as the mean \pm standard deviation (SD). Two independent groups were compared using unpaired Student's t-test, while $>2$ groups were analyzed using one-way ANOVA followed by Tukey's post hoc test using SPSS 19.0 (SPSS Inc.). P<0.05 was considered to indicate a statistically significant difference. All experiments were performed independently at least three times and the detailed times are shown in the figure legends.

\section{Results and Discussion}

Characterization of pTi implants. pTi implants were successfully manufactured by EBM technology. Representative optical images of the pTi implants are presented in Fig. 2A. The micro-CT quantitative analysis revealed the porosity of the pTi was $70.08 \pm 0.55 \%$. The pore size detected based on the SEM pictures was mainly distributed at 580-620 $\mu \mathrm{m}$ (Fig. 2B). The 

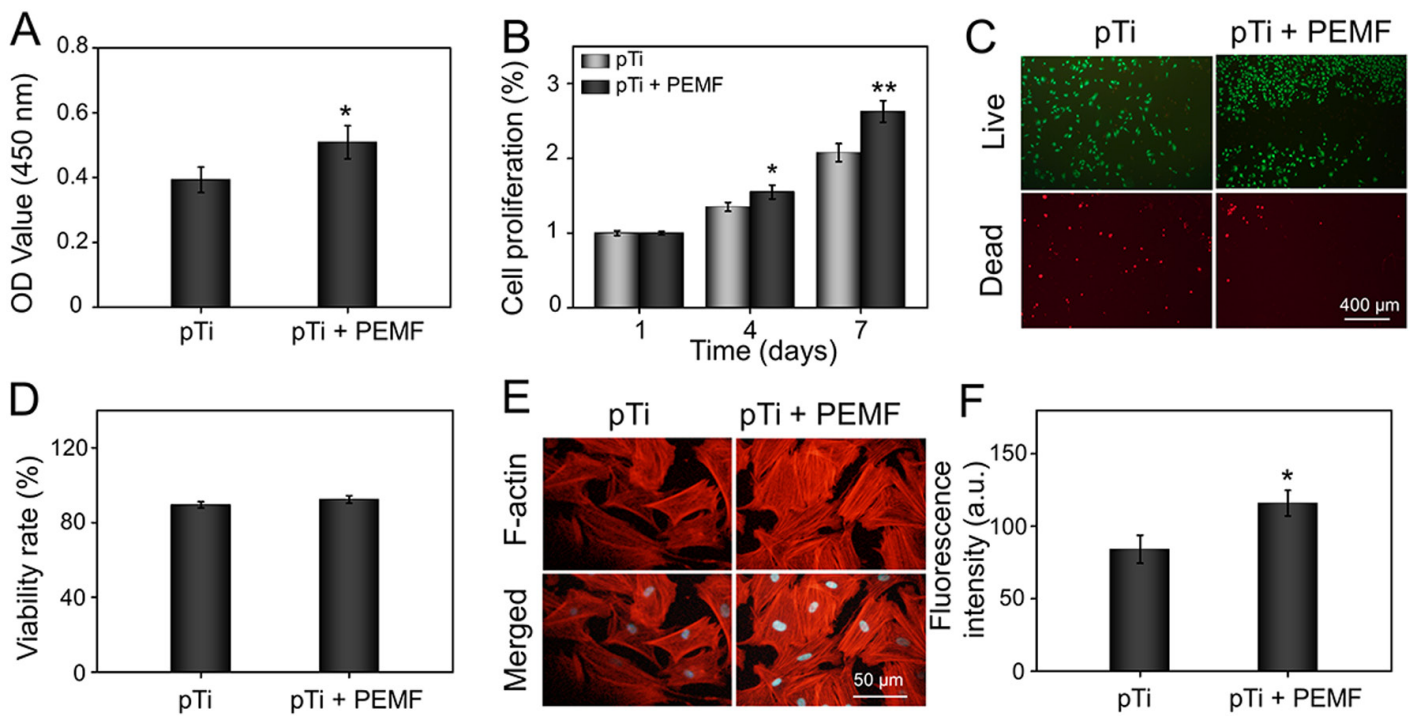

Figure 3. Cell viability of OP-BMSCs in the pTi and pTi + PEMF groups. (A) Cell attachment on the pTi scaffold following 24-h incubation. (B) Cell proliferation at day 1,4 and 7. (C) Calcein AM/PI staining of OP-BMSCs at day 3. The green signal represents live cells, whereas the red signal represents dead cells. (D) Quantitative analysis of Calcein AM/PI staining. (E) Fluorescence images of cellular morphology in the pTi group and pTi + PEMF group following 3-day culture. F-actin filaments of BMSCs were stained with rhodamine-phalloidin, whereas nuclei were stained with DAPI. (F) Quantitative analysis of F-actin. ${ }^{*} \mathrm{P}<0.05,{ }^{* *} \mathrm{P}<0.01$ vs. pTi. pTi, porous titanium; PEMF, pulse electromagnetic field; OD, optical density; a.u., arbitrary units; OP-BMSCs, osteoporosis-derived bone marrow mesenchymal stem cells; AM/PI, calcein acetoxymethyl ester/propidium Iodide.

average pore size was $600.11 \pm 7.21 \mu \mathrm{m}$ (Fig. 2C). Therefore, the actual porosity and pore size were in accordance with the pre-designed parameters (600 $\mu \mathrm{m}$ and $70 \%$, respectively).

The osseointegrative capacity of implants is usually determined by the osteoconductivity of the prosthesis, which is further bound up with the porosity, pore size and distribution of the scaffolds. The porosity of porous implants for bone tissue engineering, is expected to be $>50 \%$, especially within the scope of $65-75 \%$, which is mechanically and structurally similar to human trabecular bone. Additionally, a 300-700 $\mu \mathrm{m}$ pore size is beneficial for osteoblast adhesion, differentiation and proliferation (30). Thus, the porosity and pore size of pTi implants generated in this study were optimal for bone tissue engineering. It has been reported that the increased surface area and porosity of the implants can enhance the initial stability, bone ingrowth ability and the friction coefficient between the bone and scaffolds, thus decreasing micromotion and accelerating osseointegration after implanting in vivo (31). Furthermore, interconnected internal structure of the pTi scaffolds were favorable to oxygen and nutrient exchange, therefore improving cell growth and communication and further enhancing bone regeneration (32).

Cell attachment, proliferation, survival and morphology. Under normal conditions, the bone is continuously renewed through a coordinated progress involving complex stem cell behaviors, including adhesion, proliferation, differentiation, maturation and mineralization (33). By contrast, under the condition of osteoporosis, BMSCs present decreased ability of proliferation and osteoblastic differentiation leading to limited capacity of bone regeneration (34). External PEMF have been shown to promote cell proliferation, osteogenesis and mineralization $(21,22)$. Therefore, it was hypothesized that PEMF could be applied as a potential target to treat osteoporosis by modulating BMSC behavior. To test this hypothesis, the
OP-BMSCs harvested from the OVX rabbits were added onto the pTi and received PEMF therapy.

The osseointegration between the implants and bone tissue starts from osteogenesis-related cells adhering to the surface of the prosthesis (28). As shown in Fig. 3A, the absorbance, which stood for the number of OP-BMSCs, was significantly increased in the pTi + PEMF group compared with that in the pTi group. Moreover, cell proliferation in the different groups was evaluated using a CCK- 8 assay. In the OP-BMSCs from the pTi + PEMF group, cell proliferation was significantly increased compared with that in the pTi group on day 4, and this difference was more distinct on day 7 (Fig. 3B). OP-BMSCs seeded on the implants treated with or without PEMF were stained with Calcein AM/PI to measure cell viability. The fluorescent images demonstrated that the OP-BMSCs possessed good cell viability in the pTi and pTi + PEMF groups on day 3 (Fig. 3C). Furthermore, the viability rate of OP-BMSCs was also quantified. The results showed that the viability rates in the pTi and pTi + PEMF groups were $89.52 \pm 1.64,92.34 \pm 2.00 \%$, respectively, and there was no significant difference between the two groups (Fig. 3D). These results suggested that the pTi have good biocompatibility, and that external PEMF could promote cell attachment and proliferation, as well as and maintain viability of OP-BMSCs.

Because the morphology of the cells on the implants could dramatically influence cellular behavior, the cytoskeletal distribution of cells attached on the hydrogel was then analyzed (35). OP-BMSCs were double-stained with rhodamine-phalloidin (indicating the F-actin filament) and DAPI (indicating the nucleus) for observation. As demonstrated in Fig. 3E, the F-actin filament morphology of OP-BMSCs on the pTi implants with PEMF therapy showed better spreading and displayed more lamellipodia extensions than those on pTi scaffolds without PEMF therapy, indicating that the external PEMF could induce cell maturation (36). The intensity of 

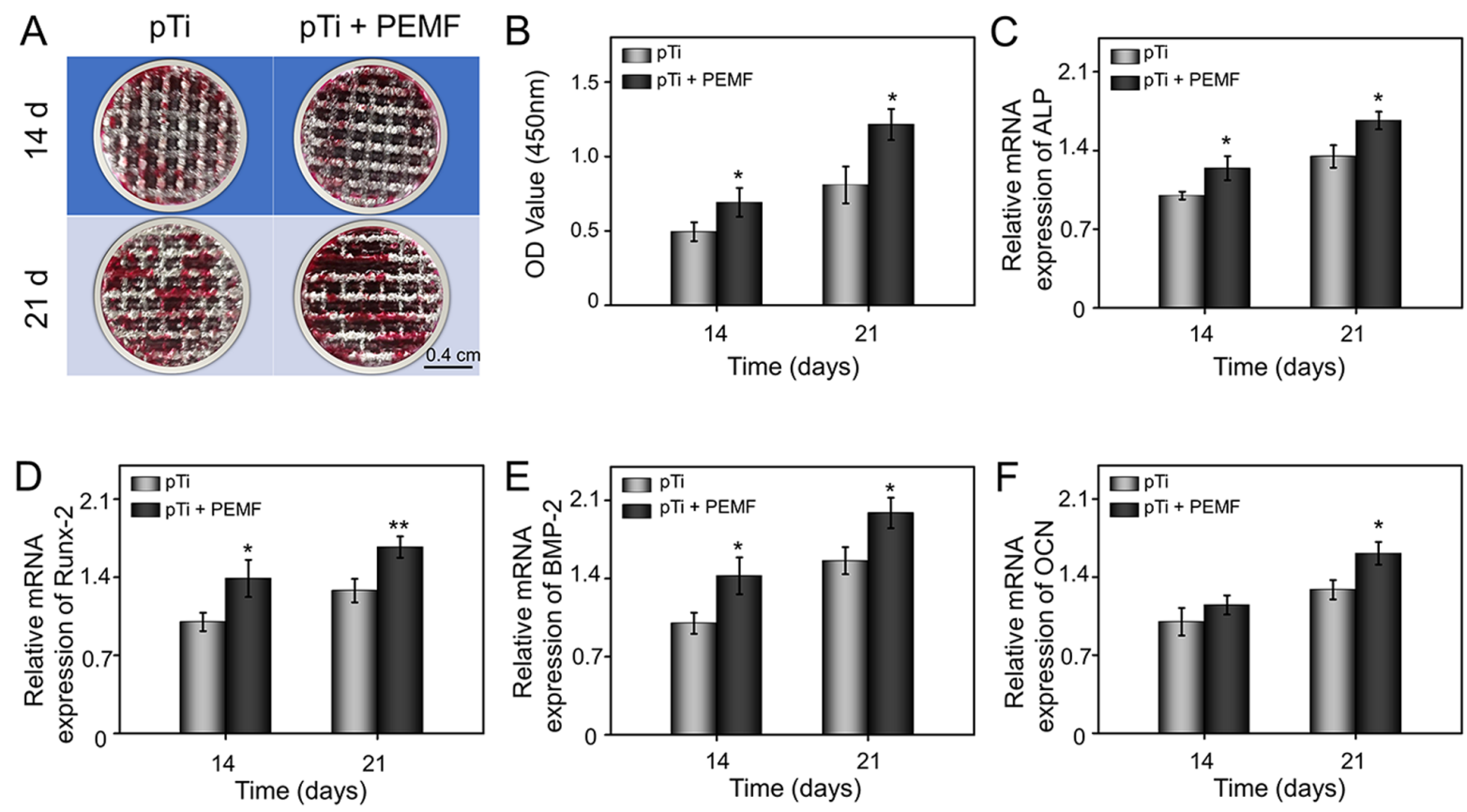

Figure 4. Osteogenic differentiation of OP-BMSCs in the pTi group and pTi + PEMF group. (A) Gross images and (B) semi-quantitative analysis of alizarin red staining after 14 and 21 days of osteogenesis induction. (C-F) mRNA expression levels of osteogenesis-related genes, ALP, RUNX-2, BMP-2, and OCN in OP-BMSCs grown on pTi or pTi + PEMF after osteogenic induction culture for 14 and 21 days. " $\mathrm{P}<0.05$, ${ }^{*} \mathrm{P}<0.01$ vs. pTi. pTi, porous titanium; PEMF, pulse electromagnetic field; OD, optical density; OP-BMSCs, osteoporosis-derived bone marrow mesenchymal stem cells; ALP, alkaline phosphatase; Runx-2; runt-related transcription factor-2; OCN, osteocalcin, BMP-2, bone morphogenetic protein-2.

F-actin on the OP-BMSCs was further quantitatively analyzed in the immunofluorescent images. As shown in Fig. 3F, OP-BMSCs in pTi + PEMF group showed increased F-actin fluorescence (1.38-fold) compared to that in the pTi group. It is well-recognized that the F-actin filament plays a fundamental role in the early maturation of BMSCs, and helps improve bone cell function by modulating cell proliferation and differentiation $(36,37)$. Therefore, PEMF therapy can significantly enhance the expression of F-actin filaments and improve cytoskeletal organization, which is beneficial for the early osteogenic differentiation of BMSCs.

Osteogenic differentiation of OP-BMSCs. In addition to cell attachment, proliferation, survival and morphology of OP-BMSCs and osteogenic differentiation are critical factors for the initiation of bone regeneration (28). In order to explore the effect of external PEMF on cell osteogenic differentiation on the pTi implants, ARS was used to assess mineralized matrix synthesis. ARS is a histological staining method for mineralization, which indicates calcium deposition. As demonstrated in Fig. 4A, calcium deposits in the pTi group were limited at day 14. However, in the pTi + PEMF group, visible calcified nodules were detected, which were more prominent on day 21. Furthermore, the semi-quantitative analysis confirmed that the cells in pTi + PEMF group exhibited increased calcium deposition compared with the pTi group at 14 and 21 days (Fig. 4B). Therefore, these results indicated that PEMF could promote the formation of mineralized matrix and further enhance the efficiency of mineralization.

In order to verify the effect of external PEMF on osteogenic differentiation of OP-BMSCs on the pTi implants at the mRNA level, multiple osteogenic differentiation markers, such as ALP, Runx-2, OCN and BMP-2, were detected using RT-qPCR. In general, ALP is marker for osteogenic differentiation, and the stimulation of ALP activity is a critical event in early osteogenesis (38). The results indicated the mRNA level of ALP was significantly increased in the pTi + PEMF group after treatment for either 14 days or 21 days, compared with that of the pTi group (Fig. 4C). Runx-2 is a indicative of early osteoblastic differentiation and can induce the expression of key osteogenic genes, such as OCN and osteopontin (39). Compared with the pTi group, the expression of Runx-2 in the pTi + PEMF group was upregulated 1.39-fold and 1.31-fold on day 14 and 21, respectively (Fig. 4D). BMP-2 is an essential osteoinductive factors and recognized to induce the differentiation of BMSCs into osteoblasts (40). Compared with pTi group, the transcriptional level of BMP-2 was upregulated significantly at day 14 and 21 in the presence of external PEMF (Fig. 4E). OCN is a bone-specific protein synthesized by osteoblasts that can enhance osteogenic maturation and bone formation; it is expressed most abundantly at the late stage of osteogenesis (41). The expression level of OCN was measured in order to evaluate the osteogenic maturation of OP-BMSCs. The expression of OCN in pTi + PEMF group did not differ significantly at day 14 , but was significantly upregulated at day 21, compared with the pTi group (Fig. 4F).

Based on the detection of ARS and quantification of osteogenic-related gene expression, it may be hypothesized that external PEMF could enhance the osteogenic differentiation of OP-BMSCs on the pTi implants. However, the mechanism through which PEMF can promote osteogenic differentiation remains unclear. For the osteogenic induction of BMSCs by PEMF, previous studies have obtained partial mechanism level explanation. Bagheri et al (42) confirmed that PEMF 
A
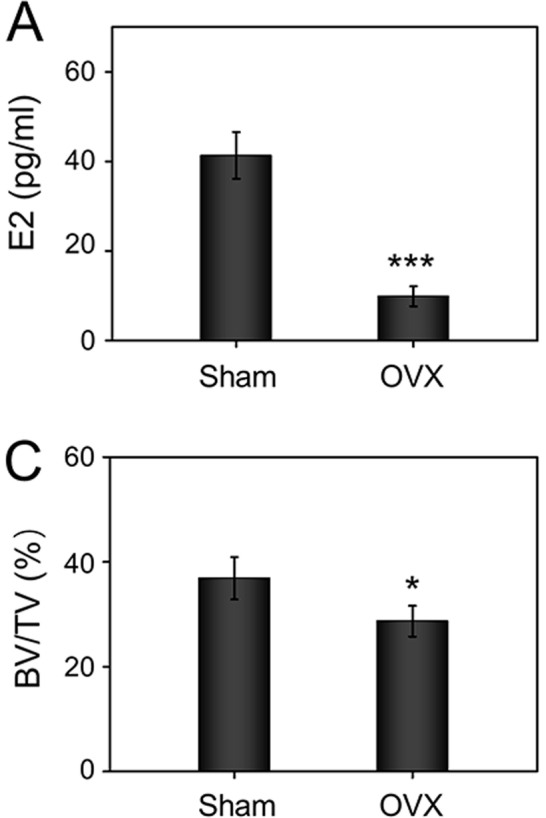

B
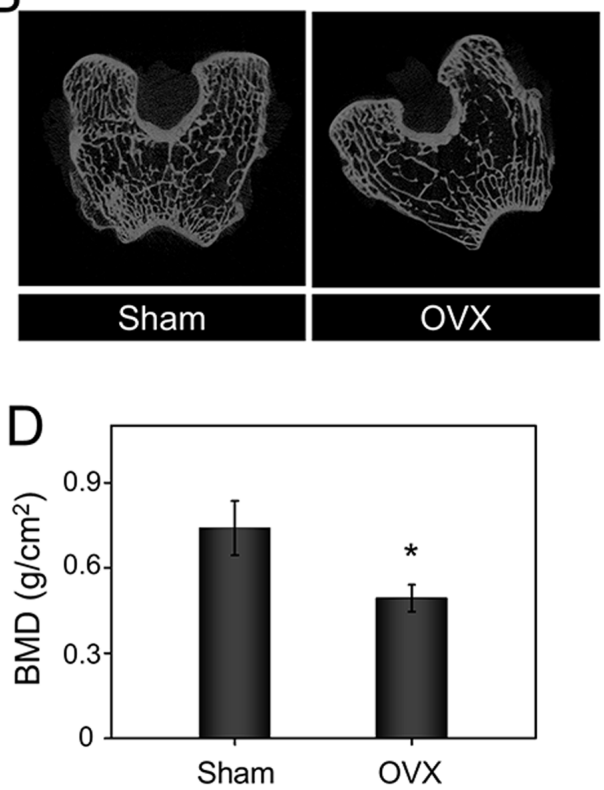

Figure 5. Establishment of the osteoporosis model 10 months after OVX. (A) Serum E2 levels. (B) Micro-CT cross-section layers of trabecular structure in distal femur. (C) Quantitative analysis of BV/TV ratio by micro-CT scanning. (D) Quantitative analysis of BMD by Micro-CT scanning. ${ }^{*}<0.05$, ${ }^{* * *} \mathrm{P}<0.001$ compared vs. sham. OVX, ovariotomy; E2, estrogen 2; CT, computed tomography; BV, bone volume; TV, tissue volume ratio; BMD, bone mineral density. pTi, porous titanium; PEMF, pulse electromagnetic field.

activated the osteogenic differentiation of BMSCs through the Notch pathway. Zhou et al (43) suggested that PEMF induced the osteogenic differentiation of BMSCs by activating Wnt/ $\beta$-catenin signaling. In addition, Selvamurugan et al (44) showed that PEMF induced the TGF- $\beta$ signaling pathway and increased the expression of microRNA-21-5p in the bone metabolism of BMSCs. Similarly, beneficial osteogenic induction effects were also observed in the present study in OP-BMSCs treated with external PEMF.

Establishment of osteoporosis model. In osteoporosis, the imbalance between bone formation and bone resorption leads to reduced bone mineral density (BMD), which improves risk of fracture and obstructed the ability of bone regeneration $(1,2)$. It has previously been reported that estrogen deficiency can result in increased bone resorption, thus impairing osteoblast function (45). The serum levels of estrogen was significantly inhibited in the OVX group compared with those of the sham group (Fig. 5A). Moreover, Micro-CT 2D slides showed the trabecular bone structure became looser and thinner in the OVX group (Fig. 5B). The statistical analysis suggested that the BMD (Fig. 5C) and BV/TV (Fig. 5D) in the OVX rabbits were significantly decreased compared with those in the sham group. Thus, these results indicated that the osteoporosis model was successfully established 10 months after bilateral OVX.

Evaluation of bone regeneration and osseointegration in vivo. Osteogenic-associated growth factors, such as BMP-2, are downregulated in the osteoporotic microenvironment (3). Furthermore, BMSCs derived from osteoporosis are few in number and deficient in osteogenic activity (4). The microenvironment with low osteogenic activity, and the imbalance between bone formation and resorption in osteoporosis state increase the risk of implants loosening and displacement after implantation. Developing novel combination therapy that promotes the osteogenic differentiation of OP-BMSCs and enhances osseointegration to decrease implant loosening and displacement remains a challenge. In order to evaluate bone regeneration and osseointegration induced by PEMF, an in vivo study was performed in OVX rabbits.

New bone regeneration in the osteoporotic bone defects was scanned using micro-CT. Reconstruction of the porous implants and surrounding bone tissues are shown in Fig. 6A. The reconstructed spatial distribution of the new bone formation revealed that with an implantation time of 6-12 weeks, bone formation on the surface of the pTi in the pTi + PEMF group were increased significantly compared with the pTi group. To quantify the bone ingrowth, the ROI scanning of the implanted region and empty defect area was carried out. The $\mathrm{BV} / \mathrm{TV}$ values of pTi and pTi + PEMF groups were 10.35 \pm 1.88 and $14.36 \pm 1.18 \%$ at 6 weeks, and $15.02 \pm 1.20$ and $20.37 \pm 1.39 \%$ at 12 weeks (Fig. 6B), respectively, consistent with the 3D reconstruction results. In parallel, pTi + PEMF group was indicated with a higher Tb.Th and Tb.N value (Fig. 6C and D) and lower Tb.Sp value (Fig. 6E) compared to that in the pTi group at 6 and 12 weeks. In general, the results of micro-CT scanning indicated that PEMF could significantly enhance bone ingrowth into the pTi implants in osteoporotic bone defects.

Good osseointegration between host bone tissues and implants involved a series of complex biological processes, including the migration and differentiation of BMSCs. The optimal prosthesis is expected to conform to the surrounding bone tissue to avoid complications including loosening and displacement after implantation, as well as recover the function of bone. In the present study, hard tissue sections were stained with Masson's stain to evaluate bone osseointegration at the bone-implant interface. As shown in Fig. 7A, improved 

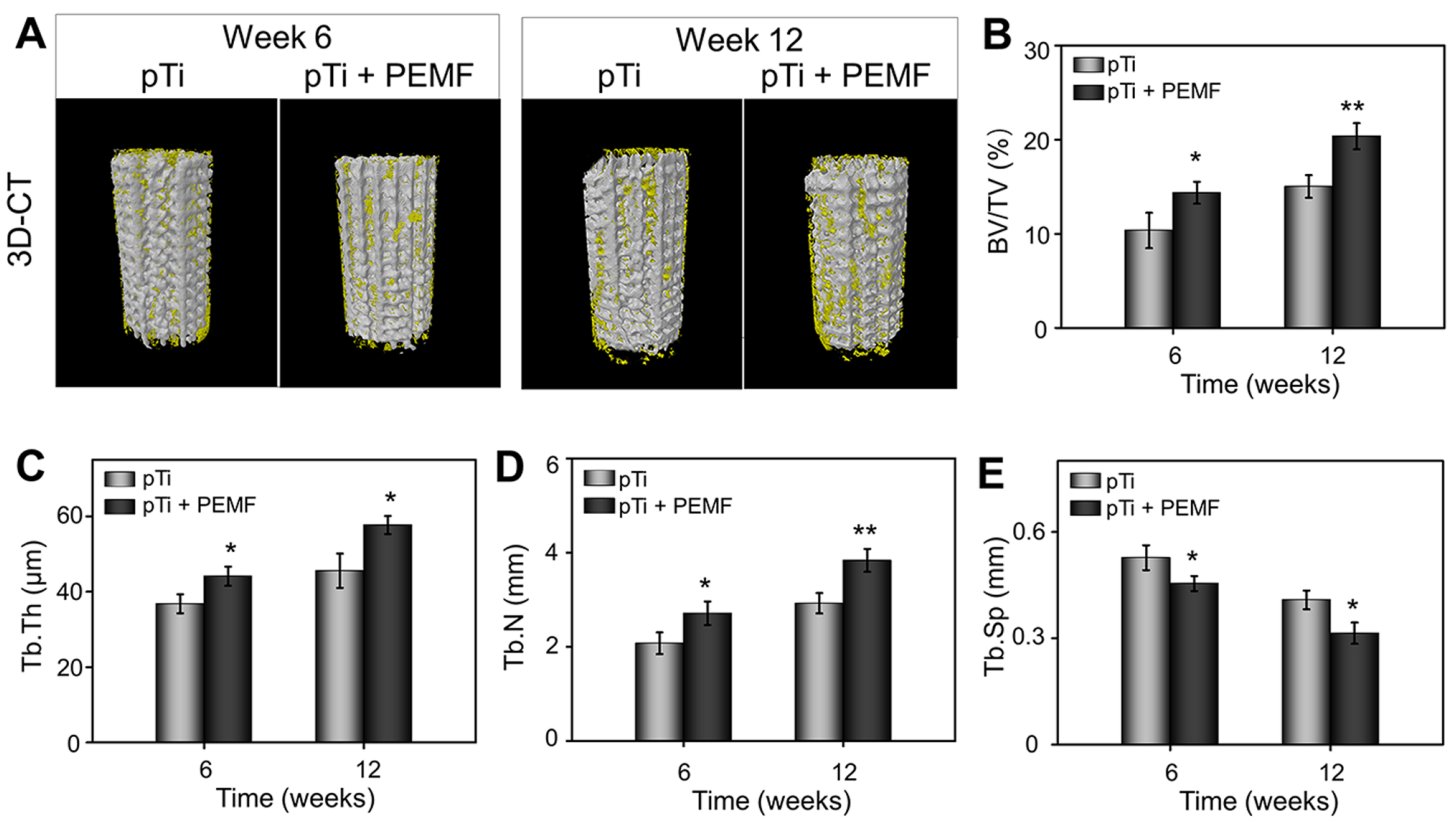

Figure 6. Micro-CT evaluation of bone regeneration in osteoporotic bone defects at 6 and 12 weeks after pTi implantation. (A) 3D reconstruction images of porous implants (white) and regenerated bone (yellow). Quantitative analysis of (B) BV/TV, (C) Tb.Th, (D) Tb.N, and (E) Tb.Sp in the pTi and pTi + PEMF groups using micro-CT. $\mathrm{P}<0.05,{ }^{* *} \mathrm{P}<0.01$ vs. pTi. pTi, porous titanium; PEMF, pulse electromagnetic field; BV, bone volume; TV, tissue volume ratio; $\mathrm{CT}$, computed tomography; Tb.N, trabecular number; Tb.Th, trabecular thickness; Tb.Sp, trabecular separation.
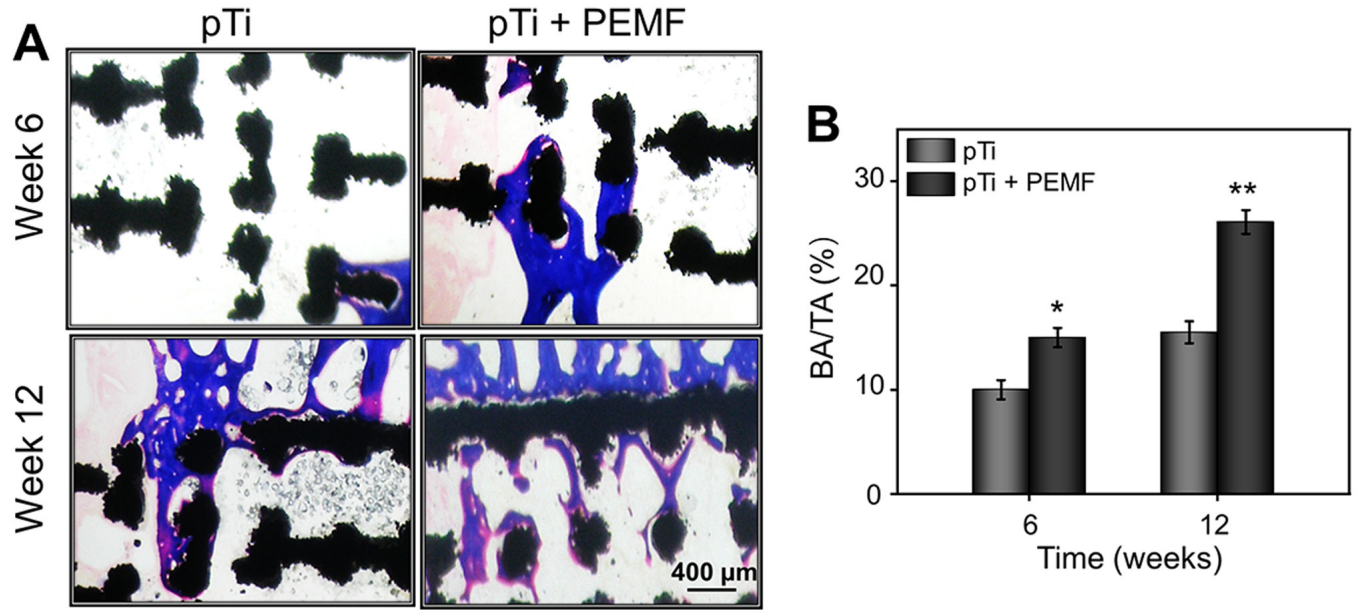

Figure 7. Histological evaluation of osseointegration. (A) Representative histological photomicrographs of Masson staining in the area of the bone defect. Black areas indicate the pTi scaffolds and the blue areas represent the bone. (B) Ratio of regenerated bone area to total defect area. ${ }^{*} \mathrm{P}<0.05$, ${ }^{* *} \mathrm{P}<0.01 \mathrm{vs}$. pTi. pTi, porous titanium; PEMF, pulse electromagnetic field; BA, bone area; TA, total area.

bone regeneration was observed between the newly formed bone and pTi implants without gaps in the pTi + PEMF group, while regenerated bone almost completely surrounded the surface of the implants along with a part of bone tissue filling the inner pores, especially after 12 weeks of PEMF treatment. Furthermore, there was a certain amount of new bone tissue around implants of the pTi group, but not as evident as that seen in the pTi + PEMF group. To calculate the ratio of bone area/total area (BA/TA), the histological pictures were analyzed using ImageJ. As shown in Fig. 7B, the BA/TA of pTi + PEMF group was significantly higher than pTi group at 6 weeks and 12 weeks, respectively.

PEMF are considered as a clinical physiotherapy that can enhancing bone regeneration (46). In the present study, pTi scaffolds were implanted into bone defects, and the addition of external PEMF led to a positive result on bone regeneration in osteoporosis models. This novel combination therapy of 3D-printed pTi and PEMF exhibited potential to promote bone regeneration and osseointegration of dental implants or artificial prostheses for patients with osteoporosis. To summarize, 3D-printed pTi implants could be customized according to desired parameters. External PEMF can effectively promote the viability and osteogenic differentiation of OP-BMSCs on the pTi surface and have great prospects for applications in bone tissue engineering. The combination of pTi and PEMF therapy resulted in improved repair effects on osteoporotic bone defects. These findings provided insight into the treatment of osseointegration under osteoporosis state. 


\section{Acknowledgements}

Not applicable.

\section{Funding}

This work was supported by the Cultivation Program from the Renji Hospital of Shanghai Jiaotong University for National Natural Science Foundation (grant no. 2018GZRPYQN06).

\section{Availability of data and materials}

The datasets used and/or analyzed during the current study are available from the corresponding author on reasonable request.

\section{Authors' contributions}

MY and WL designed the study, wrote the protocol, collected the data, performed statistical analyses and contributed to writing the manuscript. LY performed the technical work. SC helped with data collection, study design and coordinated the study. XL participated in the study design and helped to critically revise the manuscript. SQ performed study design, analysis and interpretation of data, critical revision of the manuscript and supervised the study. MY and SQ confirm the authenticity of all the raw data. All authors read and approved the final manuscript.

\section{Ethics approval and consent to participate}

All animal procedures were examined and approved by The Animal Ethics Committee of Renji Hospital of Shanghai Jiaotong University (approval no. SHJT-MRJ-2020-076).

\section{Patient consent for publication}

Not applicable.

\section{Competing interests}

The authors declare that they have no competing interests.

\section{References}

1. Lau RY and Guo X: A review on current osteoporosis research: With special focus on disuse bone loss. J Osteoporos 2011: 293808, 2011.

2. Aghebati-Maleki L, Dolati S, Zandi R, Fotouhi A, Ahmadi M, Aghebati A, Nouri M, Kazem Shakouri S and Yousefi M: Prospect of mesenchymal stem cells in therapy of osteoporosis: A review. J Cell Physiol 234: 8570-8578, 2019.

3. Boyce BF, Rosenberg E, de Papp AE and Duong LT: The osteoclast, bone remodelling and treatment of metabolic bone disease. Eur J Clin Invest 42: 1332-1341, 2012.

4. Liu W, Wang T, Yang C, Darvell BW, Wu J, Lin K, Chang J, Pan $\mathrm{H}$ and Lu WW: Alkaline biodegradable implants for osteoporotic bone defects-importance of microenvironment $\mathrm{pH}$. Osteoporos Int 27: 93-104, 2016.

5. Brandi ML: Healing of the bone with anti-fracture drugs. Expert Opin Pharmacother 14: 1441-1447, 2013.

6. Russell LA: Osteoporosis and orthopedic surgery: Effect of bone health on total joint arthroplasty outcome. Curr Rheumatol Rep 15: 371, 2013
7. Bottai V, Dell'Osso G, Celli F, Bugelli G, Cazzella N, Cei E, Guido G and Giannotti S: Total hip replacement in osteoarthritis: The role of bone metabolism and its complications. Clin Cases Miner Bone Metab 12: 247-250, 2015.

8. Mei S, Wang H, Wang W, Tong L, Pan H, Ruan C, Ma Q, Liu M, Yang H, Zhang L, et al: Antibacterial effects and biocompatibility of titanium surfaces with graded silver incorporation in titania nanotubes. Biomaterials 35: 4255-4265, 2014

9. Zhang L, Le Coz-Botrel R, Beddoes C, Sjöström T and Su B: Gelatin freeze casting of biomimetic titanium alloy with anisotropic and gradient pore structure. Biomed Mater 12: 015014, 2017.

10. Vladescu A, Vranceanu DM, Kulesza S, Ivanov AN, Bramowicz M, Fedonnikov AS, Braic M, Norkin IA, Koptyug A, Kurtukova MO, et al: Influence of the electrolyte's $\mathrm{pH}$ on the properties of electrochemically deposited hydroxyapatite coating on additively manufactured Ti64 alloy. Sci Rep 7: 16819, 2017.

11. Chudinova EA, Surmeneva MA, Timin AS, Karpov TE, Wittmar A, Ulbricht M, Ivanova A, Loza K, Prymak O, Koptyug A, et al: Adhesion, proliferation, and osteogenic differentiation of human mesenchymal stem cells on additively manufactured Ti6Al4V alloy scaffolds modified with calcium phosphate nanoparticles. Colloids Surf B Biointerfaces 176: 130-139, 2019.

12. Amin Yavari S, van der Stok J, Chai YC, Wauthle R, Tahmasebi Birgani Z, Habibovic P, Mulier M, Schrooten J, Weinans $\mathrm{H}$ and Zadpoor AA: Bone regeneration performance of surface-treated porous titanium. Biomaterials 35: 6172-6181, 2014.

13. Nune KC, Kumar A, Murr LE and Misra RD: Interplay between self-assembled structure of bone morphogenetic protein-2 (BMP-2) and osteoblast functions in three-dimensional titanium alloy scaffolds: Stimulation of osteogenic activity. J Biomed Mater Res A 104: 517-532, 2016.

14. Pan S, Yin J, Yu L, Zhang C, Zhu Y, Gao Y and Chen Y: 2D MXene-Integrated 3D-Printing scaffolds for augmented osteosarcoma phototherapy and accelerated tissue reconstruction. Adv Sci (Weinh) 7: 1901511, 2019.

15. Dimitriou R, Jones E, McGonagle D and Giannoudis PV: Bone regeneration: Current concepts and future directions. BMC Med 9: 66, 2011.

16. Wang Z, Wang C, Li C, Qin Y, Zhong L, Chen B, Li Z, Liu H, Chang $\mathrm{F}$ and Wang $\mathrm{J}$ : Analysis of factors influencing bone ingrowth into three-dimensional printed porous metal scaffolds: A review. J Alloy Compound 717: 271-285, 2017.

17. Bai H, Cui Y, Wang C, Wang Z, Luo W, Liu Y, Leng Y, Wang J, Li Z and Liu H: 3D printed porous biomimetic composition sustained release zoledronate to promote osteointegration of osteoporotic defects. Mater Des 189: 108513, 2020.

18. Ye D, Xu Y, Wang G, Feng X, Fu T, Zhang H, Jiang L and Bai Y: Thermal effects of $2450 \mathrm{MHz}$ microwave exposure near a titanium alloy plate implanted in rabbit limbs. Bioelectromagnetics 36 : 309-318, 2015.

19. Vayron R, Nguyen VH, Lecuelle B, Albini Lomami H, Meningaud JP, Bosc R and Haiat G: Comparison of resonance frequency analysis and of quantitative ultrasound to assess dental implant osseointegration. Sensors (Basel) 18: 1397, 2018.

20. Liang H, Liu X, Pi Y, Yu Q, Yin Y, Li X, Yang Y and Tian J: 3D-Printed $\beta$-tricalcium phosphate scaffold combined with a pulse electromagnetic field promotes the repair of skull defects in rats. ACS Biomater Sci Eng 5: 5359-5367, 2019.

21. Liu C, Yu J, Yang Y, Tang X, Zhao D, Zhao W and Wu H: Effect of $1 \mathrm{mT}$ sinusoidal electromagnetic fields on proliferation and osteogenic differentiation of rat bone marrow mesenchymal stromal cells. Bioelectromagnetics 34: 453-464, 2013.

22. Tsai MT, Chang WH, Chang K, Hou RJ and Wu TW: Pulsed electromagnetic fields affect osteoblast proliferation and differentiation in bone tissue engineering. Bioelectromagnetics 28: 519-528, 2007.

23. Wang P, Liu J, Yang Y, Zhai M, Shao X, Yan Z, Zhang X, Wu Y, Cao L, Sui B, et al: Differential intensity-dependent effects of pulsed electromagnetic fields on RANKL-induced osteoclast formation, apoptosis, and bone resorbing ability in RAW264.7 cells. Bioelectromagnetics 38: 602-612, 2017.

24. Adie S, Harris IA, Naylor JM, Rae H, Dao A, Yong S and Ying V: Pulsed electromagnetic field stimulation for acute tibial shaft fractures: A multicenter, double-blind, randomized trial. J Bone Joint Surg Am 93: 1569-1576, 2011.

25. Bagnato GL, Miceli G, Marino N, Sciortino D and Bagnato GF: Pulsed electromagnetic fields in knee osteoarthritis: A double blind, placebo-controlled, randomized clinical trial. Rheumatology (Oxford) 55: 755-762, 2016. 
26. Yin Y, Chen P, Yu Q, Peng Y, Zhu Z and Tian J: The effects of a pulsed electromagnetic field on the proliferation and osteogenic differentiation of human adipose-derived stem cells. Med Sci Monit 24: 3274-3282, 2018.

27. Qiao S, Sheng Q, Li Z, Wu D, Zhu Y,Lai HC and Gu Y: 3D-printed Ti6Al4V scaffolds coated with freeze-dried platelet-rich plasma as bioactive interface for enhancing osseointegration in osteoporosis. Mater Des 194: 108825, 2020.

28. Bai H, Zhao Y, Wang C, Wang Z, Wang J, Liu H, Feng Y, Lin Q, $\mathrm{LiZ}$ and Liu H: Enhanced osseointegration of three-dimensional supramolecular bioactive interface through osteoporotic microenvironment regulation. Theranostics 10: 4779-4794, 2020.

29. Livak KJ and Schmittgen TD: Analysis of relative gene expression data using real-time quantitative PCR and the 2(-Delta Delta C(T)) method. Methods 25: 402-408, 2001.

30. Li Z, Wang C, Li C, Wang Z, Yang F, Liu H, Qin Y and Wang J: What we have achieved in the design of $3 \mathrm{D}$ printed metal implants for application in orthopedics? Personal experience and review. Rapid Prototyp J 24: 1365-1379, 2018.

31. Raphel J, Holodniy M, Goodman SB and Heilshorn SC: Multifunctional coatings to simultaneously promote osseointegration and prevent infection of orthopaedic implants. Biomaterials 84: 301-314, 2016.

32. Kumar A, Nune KC and Misra RDK: Design and biological functionality of a novel hybrid Ti-6Al-4V/hydrogel system for reconstruction of bone defects. J Tissue Eng Regen Med 12: 1133-1144, 2018

33. Liang C, Peng S, Li J, Lu J, Guan D, Jiang F, Lu C, Li F, He X, Zhu $\mathrm{H}$, et al: Inhibition of osteoblastic Smurf1 promotes bone formation in mouse models of distinctive age-related osteoporosis. Nat Commun 9: 3428, 2018

34. Zhang X, Zhu Y, Cao L, Wang X, Zheng A, Chang J, Wu J, Wen J, Jiang X, Li H and Zhang Z: Alginate-aker injectable composite hydrogels promoted irregular bone regeneration through stem cell recruitment and osteogenic differentiation. J Mater Chem B 6: 1951-1964, 2018

35. Feng YF, Wang L, Zhang Y, Li X, Ma ZS, Zou JW, Lei W and Zhang ZY: Effect of reactive oxygen species overproduction on osteogenesis of porous titanium implant in the present of diabetes mellitus. Biomaterials 34: 2234-2243, 2013.

36. Zhao Y, Wang Z, Jiang Y, Liu H, Song S, Wang C, Li Z, Yang Z, Liu H, Wang J, Yang B and Lin Q: Biomimetic composite scaffolds to manipulate stem cells for aiding rheumatoid arthritis management. Adv Funct Mater 29: 1807860, 2019.

37. Yi H, Ur Rehman F, Zhao C, Liu B and He N: Recent advances in nano scaffolds for bone repair. Bone Res 4: 16050, 2016.
38. Lee BN, Hong JU, Kim SM, Jang JH, Chang HS, Hwang YC, Hwang IN and Oh WM: Anti-inflammatory and osteogenic effects of calcium silicate-based root canal sealers. J Endod 45: 73-78, 2019

39. Sivashanmugam A, Charoenlarp P, Deepthi S, Rajendran A, Nair SV, Iseki S and Jayakumar R: Injectable shear-thinning $\mathrm{CaSO}(4) / F G F-18-I n c o r p o r a t e d ~ C h i t i n-P L G A$ hydrogel enhances bone regeneration in mice cranial bone defect model. ACS Appl Mater Interfaces 9: 42639-42652, 2017.

40. Rashdan NA, Sim AM, Cui L, Phadwal K, Roberts FL, Carter R, Ozdemir DD, Hohenstein P, Hung J, Kaczynski J, et al: Osteocalcin regulates arterial calcification via altered wnt signaling and glucose metabolism. J Bone Miner Res 35: 357-367, 2020.

41. Ho SS, Vollmer NL, Refaat MI, Jeon O, Alsberg E, Lee MA and Leach JK: Bone morphogenetic protein-2 promotes human mesenchymal stem cell survival and resultant bone formation when entrapped in photocrosslinked alginate hydrogels. Adv Healthc Mater 5: 2501-2509, 2016.

42. Bagheri L, Pellati A, Rizzo P, Aquila G, Massari L, De Mattei M and Ongaro A: Notch pathway is active during osteogenic differentiation of human bone marrow mesenchymal stem cells induced by pulsed electromagnetic fields. J Tissue Eng Regen Med 12: 304-315, 2018.

43. Zhou YJ, Wang P, Chen HY, Liu C, Ji QD, Yang XT, Gao Q and He CQ: Effect of pulsed electromagnetic fields on osteogenic differentiation and Wnt/ $\beta$-catenin signaling pathway in rat bone marrow mesenchymal stem cells. Sichuan Da Xue Xue Bao Yi Xue Ban 46: 347-353, 2015 (In Chinese).

44. Selvamurugan N, He Z, Rifkin D, Dabovic B and Partridge NC: Pulsed electromagnetic field regulates MicroRNA 21 expression to activate TGF- $\beta$ signaling in human bone marrow stromal cells to enhance osteoblast differentiation. Stem Cells Int 2017: 2450327, 2017.

45. Rachner TD, Khosla S and Hofbauer LC: Osteoporosis: Now and the future. Lancet 377: 1276-1287, 2011.

46. Ehnert S, Schröter S, Aspera-Werz RH, Eisler W, Falldorf K, Ronniger M and Nussler AK: Translational insights into extremely low frequency pulsed electromagnetic fields (ELF-PEMF) for bone regeneration after trauma and orthopedic surgery. J Clin Med 8: 2028, 2019.

This work is licensed under a Creative Commons Attribution-NonCommercial-NoDerivatives 4.0 International (CC BY-NC-ND 4.0) License. 